\title{
Nonlinear Observation in Fuel Cell Systems: A Comparison between Disturbance Estimation and High-Order Sliding-Mode Techniques
}

\author{
Julio Luna ${ }^{\mathrm{a}, *}$, Elio Usai ${ }^{\mathrm{b}}$, Attila Husar ${ }^{\mathrm{a}}$, Maria Serra ${ }^{\mathrm{a}}$ \\ ${ }^{a}$ Institut de Robòtica i Informàtica Industrial (CSIC-UPC). C/Llorens i Artigas 4-6. 08028 Barcelona, Spain \\ ${ }^{b}$ Università di Cagliari. Dipartimento di Ingegneria Elettrica ed Elettronica (DIEE), Piazza d'Armi, 09123 Cagliari, Italy
}

\begin{abstract}
This paper compares two Nonlinear Distributed Parameter Observers (NDPO) for the observation of a Proton Exchange Membrane Fuel Cell (PEMFC). Both NDPOs are based on the discretisation of distributed parameters models and they are used to estimate the state profile of gas concentrations in the anode and cathode gas channels of the PEMFC, giving detailed information about the internal conditions of the system. The reaction and water transport flow rates from the membrane to the channels are uncertainties of the observation problem and they are estimated throughout all the length of the PEMFC without the use of additional sensors. The first observation approach is a Nonlinear Disturbance Observer (NDOB) for the estimation of the disturbances in the NDPO. In the second approach, a novel implementation of a High-Order Sliding-Mode (HOSM) observer is developed to estimate the true value of the states as well as the reaction terms. The proposed observers are tested and compared through a simulation example at different operating points and their performance and robustness is analysed over a given case study, the New European Driving Cycle.
\end{abstract}

Keywords: PEMFC, distributed parameter system, nonlinear observer, disturbance observer, sliding-mode

\section{Introduction}

Hydrogen as an energy vector is an interesting alternative to support the increasingly renewable energy landscape of the future. In this context, PEMFCs, which use hydrogen as fuel, are compelling for mass market applications such as automotive and stationary Combined Heat and Power (CHP) systems. Extensive research efforts are being dedicated to improve efficiency, reduce degradation and decrease production costs of this technology.

The efficiency and degradation of a PEMFC system is greatly related to the internal operating conditions. In the automatic control field, new observation $[1,2]$, diagnosis $[3,4,5]$ and control $[6,7,8]$ techniques are being developed to improve the operating conditions of PEMFC systems. Temperature, gas concentrations, current density and water content in the different layers, among others, affect the performance of the electrochemical system. On the other hand, PEMFCs lifetime is reduced drastically when the fuel cell is subject to unexpected load changes and global or local reactant starvation occurs [9]. Global starvation can be avoided measuring the current of the system and manipulating the input fuel and oxidant reactant flows. Nonetheless, to avoid local starvation it is necessary to know the internal concentration values in the fuel cell. Since PEMFCs are hermetic systems, the measurement of the internal variables is a demanding or even impossible task from the technological point of view and commercial systems are not prepared to measure the internal conditions. However, using nonlinear state observation with disturbance and unknown input reconstruction it is possible to estimate the internal variables without using additional sensors.

Regarding nonlinear observation in PEMFC systems, a limited number of works have been published. A critical parameter to be estimated in a PEMFC is the membrane water content $[10,11]$, since this variable has an important effect on the PEMFC efficiency and overall system performance. Other works present the nonlinear observation of the inlet hydrogen and oxygen flows through the measurement of the input and output manifold pressures of the PEMFC $[12,13]$. Measuring the compressor rotational speed and the pressures in the cathode inlet and outlet manifolds, the oxygen excess ratio in the fuel cell is observed in [14] and its observed value is used in a feedback control loop.

In this paper, two nonlinear observation techniques are proposed to estimate the PEMFC concentration full profiles in the presence of uncertainties (reaction rates and water transport terms). The first approach is based on an observation methodology that was applied to a PEMFC in [15] under the assumption that the reaction terms were measured variables. The main improvement in this paper with respect to [15] is to remove the

*Corresponding author. Tel: +349340158 05 - Fax: +34934015750.

Email address: jluna@iri.upc.edu (Julio Luna) 
unrealistic assumption that considers the reaction terms as measured variables and recognise them as internal disturbances of the model. This adds additional uncertainty to the observation procedure. To cope with it, in this paper a disturbance observer is designed to estimate the reaction terms.

The second approach is a novel NDPO design based on a quasi-continuous Sliding Mode (SM) method [16]. Both approaches present robustness and proper convergence rate capabilities. However, some differences have been found and they are analysed in this paper. Both observation techniques are studied under a case study, the New European Driving Cycle (NEDC). This highly dynamic case study will show how both observation techniques behave under more realistic driving conditions.

Summarising, the main contributions of this paper are:

(i) The extension of the NDPO in [15] by means of a super-twisting Nonlinear Disturbance Observer (NDOB) in [15] to estimate both the states and the unknown disturbances in finite time

(ii) The development of a novel HOSM observer based on quasi-continuous SM techniques to observe both the nonlinear states and the unmatched disturbances of the PEMFC

(iii) The comparison of the previous observers using the NEDC as a simulation scenario to determine the best approach for the future synthesis of advanced controllers aimed at improving the efficiency and life expectancy of the PEMFC system

The paper is organised as follows. In Section 2, the general system description and the simulation model of the system, based on distributed parameters, are introduced. The observation problem and the primary observer are presented in Section 3. In Section 4 the NDPO with NDOB is developed. The HOSM observer is formulated and explained in Section 5. Simulation results for the two proposed observers are presented and compared in detail in Section 6. Finally, Section 7 summarizes the overall results of this paper and proposes some research lines for future work.

\section{Nomenclature}

Throughout this paper, spatially distributed systems are treated, denoting the spatial variables as $x, y$ and $z$. Subscripts $i$ and $j$ are associated to the reactant and discretisation volume respectively. For instance, $c_{i, j}$ refers to the concentration value of the $i$-th gas at the $j$ discretisation volume. The superscripts are associated to an element of the PEMFC, being $A$ the anode, $C$ the cathode and $M$ the membrane. Moreover, the superscript $k$ is used for equations valid both for the anode and cathode sides. All column vectors are denoted by bold style, e.g. x. Matrices are denoted by bold upper case, e.g., A. Scalars are denoted by non-bold style, e.g., $B$. The set of real numbers is denoted by $\mathbb{R}$. Observed variables are denoted by the caret symbol, e.g., $\hat{x}$.

\section{Simulation model}

\subsection{System description}

This paper is focused on a single-channel PEMFC. See Figure 1 for the overview of the PEMFC and the reference coordinate system employed. The modelled single channel PEMFC [17] has a channel length $(L)$ of $0.4 \mathrm{~m}$ and a channel width $\left(L_{x}\right)$ of $1 \times 10^{-3} \mathrm{~m}$. The total active area is equal to $0.4 \times 10^{-3} \mathrm{~m}^{2}$.

The simulation model considers the gas channels, Gas Diffusion Layers (GDL), Catalyst Layers (CL) and the electrolyte membrane of the PEMFC [17]. Figure 1 (bottom left) shows the modelling domain for the simulation model. All modelling variables and parameters are collected in Table 1.

\subsection{Model assumptions}

A $1+1 \mathrm{D}$ or quasi-two dimensional parameters model [17] is used to simulate the PEMFC in this paper. Some assumptions have been considered for its implementation. For instance, the electrical conductivity is related proportionally to the membrane water content of the PEMFC. Furthermore, the gas flow dynamics in the anode and cathode gas channels are described by partial derivatives along the z-direction. Chemical gradients [18] drive the hydrogen, oxygen and water molecules along the y-axis direction in the diffusion layers, catalyst layers and membrane. It is considered that the membrane can retain water in its interior, however, the swelling effect is not considered and the absorption and desorption effects are not modelled. The study assumptions include isothermal conditions. 


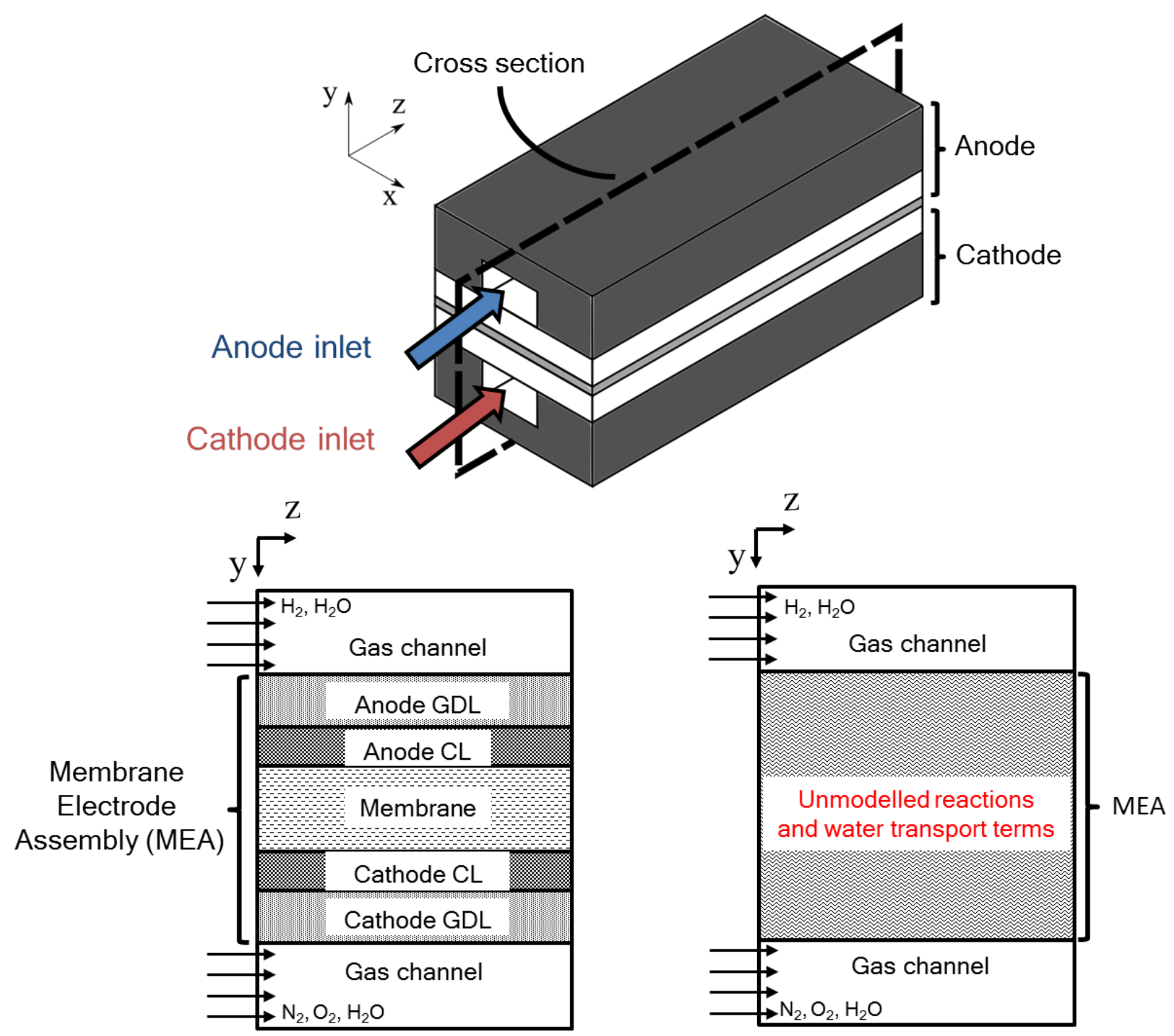

Figure 1: Single-channel PEMFC representation (top); simulation (bottom left) and observer (bottom right) modelling domains 
Table 1: Physical properties and constants

\begin{tabular}{|c|c|c|}
\hline Parameter & Description & Units \\
\hline$c_{i}$ & Concentration of $i$-th gas & $\mathrm{mol} \mathrm{m}^{-3}$ \\
\hline$D_{i}$ & Diffusion coefficient of $i$-th gas & $\mathrm{m}^{2} \mathrm{~s}^{-1}$ \\
\hline$E_{r}$ & Ideal potential voltage & $\mathrm{V}$ \\
\hline$F$ & Faraday constant & $\mathrm{C} \mathrm{mol}^{-} 1$ \\
\hline$f^{V}$ & Surface enlargement factor & - \\
\hline$i$ & Current density & $\mathrm{A} \mathrm{m}^{-2}$ \\
\hline$i_{0}$ & Exchange current density & $\mathrm{A} \mathrm{m}^{-2}$ \\
\hline$K$ & Pressure drop coefficient & $\mathrm{m}^{2} \mathrm{~s}^{-1} \mathrm{~Pa}^{-1}$ \\
\hline$L$ & Fuel cell length & $\mathrm{m}$ \\
\hline$L_{x}$ & Gas channel width & $\mathrm{m}$ \\
\hline$L_{z}$ & Gas channel depth & $\mathrm{m}$ \\
\hline$\dot{n}_{i, i n}$ & Inlet molar flux of $i$-th gas & $\mathrm{mol} \mathrm{m} \mathrm{m}^{-2} \mathrm{~s}^{-1}$ \\
\hline$\dot{n}_{i}$ & $\mathrm{y}$-direction flux of $i$-th gas & $\mathrm{mol} \mathrm{m} \mathrm{m}^{-2} \mathrm{~s}^{-1}$ \\
\hline$n$ & Discretisation volumes & - \\
\hline$p$ & Pressure & $\mathrm{Pa}$ \\
\hline$R$ & Gas constant & $\mathrm{J} \mathrm{mol}^{-1} \mathrm{~K}^{-1}$ \\
\hline$r$ & Reaction rate & $\mathrm{mol} \mathrm{m} \mathrm{m}^{-2} \mathrm{~s}^{-1}$ \\
\hline$T$ & Temperature & $\mathrm{K}$ \\
\hline$V$ & Electrical potential & $\mathrm{V}$ \\
\hline$v$ & Flow velocity & $\mathrm{m} \mathrm{s}^{-1}$ \\
\hline$\alpha$ & Heat transfer coefficient & $\mathrm{W} \mathrm{m}^{-2} \mathrm{~K}^{-1}$ \\
\hline$\Delta G_{0}$ & Gibbs activation energy & $\mathrm{J} \mathrm{mol}^{-1}$ \\
\hline$\Delta z$ & Discretisation length & $\mathrm{m}$ \\
\hline$\delta$ & $\mathrm{y}$-axis thickness & $\mathrm{m}$ \\
\hline
\end{tabular}

\subsection{PEMFC model}

\subsubsection{Electrochemical model}

The fuel cell electrical power is expressed as

$$
P_{f c, e l e c}(t)=V_{f c}(t) I_{f c}(t)
$$

being $V_{f c}$ the total fuel cell stack voltage and $I_{f c}$ the total current delivered by the PEMFC.

The single-cell voltage is modelled with the Butler-Volmer equation [19]

$$
V_{f c, \text { cell }}(t)=E_{r}-\frac{R T}{\alpha 2 F}\left[\ln \left(\frac{i(t)}{i_{0}(t)}\right)-\ln \left(\frac{p_{O_{2}}^{C}(t)}{p_{O_{2}, r e f}^{C}(t)}\right)\right]-i(t) R_{o h m}
$$

being $i$ the demanded current density, $E_{r}$ the ideal potential voltage of the fuel cell, $\alpha$ the cathode heat transfer coefficient, $R_{o h m}$ the internal resistance of the membrane, $p_{\mathrm{O}_{2}}^{C}$ the oxygen pressure and $p_{\mathrm{O}_{2}, \text { ref }}^{C}$ the oxygen pressure reference at the cathode CL.

The exchange current density $i_{0}$ in Eq. (2) describes the electrochemical reaction kinetics of the PEMFC and is expressed as follows:

$$
i_{0}(t)=\frac{1}{2} F \dot{n}_{H_{2}}^{A}(t)=\frac{1}{2} F r^{A}(t),
$$

where the anodic reaction rate $r^{A}[17]$ is

$$
r^{A}(t)=f^{V} \frac{i_{0}^{A}}{2 F}\left[\exp (\Omega(t)) \frac{p_{H_{2}}^{A}(t)}{p_{H_{2}, r e f}^{A}}-1\right]
$$


being

$$
\Omega(t)=\frac{2 F}{R T}\left(\Delta V^{A}(z, t)-\Delta V_{r e f}^{A}(z)\right)
$$

\subsubsection{Gas flow model}

The dynamics of the gas flows in the channels are described by the following mass balance equations:

$$
\begin{aligned}
\frac{\partial c_{i}(t)}{\partial t} & =\frac{\partial}{\partial z}\left(v(t) c_{i}(t)\right)-\frac{\dot{n}_{i}}{\delta} \\
v(t) & =-K \frac{\partial p(t)}{\partial z} \\
p(t) & =R T \sum_{i} c_{i}(t)
\end{aligned}
$$

where subscript $i$ stands for the reactant, namely $i=\mathrm{H}_{2}$ is the hydrogen index and $i=\mathrm{H}_{2} \mathrm{O}$ is the index of the vapour water in the anode side. At the cathode side $i=N_{2}$ denotes the nitrogen, $i=O_{2}$ the oxygen and $i=\mathrm{H}_{2} \mathrm{O}$ the vapour water. The velocity, pressure and concentration values of the gases depend on the position along the $z$-direction as expressed by the spatial partial derivatives. Reaction and water molar transports $\dot{n}_{i}$ are perpendicular to the gas channels in the y-direction.

Hydrogen, oxygen and water diffuse from the gass channels through the GDLs in the y-direction due to the gradient of concentration that appears between the gas channels and the CLs. The diffusion model in the GDLs of the PEMFC follows Fick's second law of difussion [20]

$$
\frac{\partial c_{i}(t)}{\partial t}=D_{i} \frac{\partial^{2} c_{i}(t)}{\partial y^{2}}
$$

with $D_{i}$ being the diffusion coefficient of the $i$-th gas species through the GDLs, excluding the nitrogen which does not react. The values of the concentrations depend on the position along the $y$-direction.

In the CLs the electrochemical reactions, which are modelled as lumped parameters, take place. The reacted y-direction molar fluxes are function of the anodic and cathodic reaction rates.

\subsubsection{Water transport model}

It is assumed that there is no mass storage capacity at the GDLs, therefore, it can be expressed that the water transport from the anode gas channel is equal to the water transport towards the membrane

$$
\dot{n}_{H_{2} O}^{A}(t)=\dot{n}_{H_{2} O}^{A M}(t)
$$

where the superscript $A M$ denotes that it is a flux exiting from the anode side and entering into the electrolyte membrane.

The water from the channel to the catalyst layer is the difference between the water from the channel to the membrane and the water generated by the reaction

$$
\dot{n}_{H_{2} \mathrm{O}}^{C}(t)=\dot{n}_{\mathrm{H}_{2} \mathrm{O}}^{C M}(t)-2 \dot{n}_{\mathrm{O}_{2}}^{C}(t)=\dot{n}_{\mathrm{H}_{2} \mathrm{O}}^{C M}(t)-r^{C}(t)
$$

being $r^{C}$ the cathodic reaction rate which is expressed as

$$
{ }^{C}(t)=\frac{R T}{\alpha 2 F}\left[\ln \left(\frac{i(t)}{i_{0}^{C}(t)}\right)-\ln \left(\frac{p_{O_{2}}^{C}(t)}{p_{O_{2}, r e f}^{C}(t)}\right)\right] .
$$

\subsubsection{Membrane model}

The membrane layer includes a complete water transport model [18]. Moreover, the membrane water content $(\Lambda)$ of the PEMFC is included in the model as the relation between the number of water moles and the moles of polymer in the membrane. The isotherms to compute the water content of the membrane at each discretisation volume are given in [18].

The water transport mechanisms that are modelled in the membrane are the electro-osmotic drag (EOD) and the back diffusion (BD). These two mechanisms are modelled with the following equation [17]:

$$
\dot{n}_{H_{2} O, j}(t)=-\frac{t_{w}\left(\Lambda_{j}\right) \kappa\left(\Lambda_{j}\right)}{F^{2}} \nabla_{+}(t)-\frac{D_{w}\left(\Lambda_{j}\right) c_{H_{2} O, j}^{M}}{R T} \nabla_{H_{2} O}(t),
$$

being $D_{w}$ the self-diffusion coefficient and $t_{w}$ a humidity-dependent transport number. $\nabla_{+}$and $\nabla_{H_{2} O}$ are the proton and water chemical potentials respectively [18]. 


\section{Discretised model derivation}

The NDPOs developed in this paper focus on the estimation of the full concentration profile of each one of the $i$-th gas species in the anode and cathode gas channels, denoted by $c_{i}$. The observation of these variables will allow to determine local starvation points caused by low fuel and oxidant concentration values in the fuel cell.

For nonlinear models with lumped parameters, the state space representation can be written as two functions of the $m$ states, $p$ inputs and $q$ outputs

$$
\begin{aligned}
& \dot{\mathbf{x}}(t)=\mathbf{f}(t, \mathbf{x}(t), \mathbf{u}(t)) \\
& \mathbf{y}(t)=\mathbf{h}(t, \mathbf{x}(t), \mathbf{u}(t)),
\end{aligned}
$$

where $\mathbf{x} \in \mathbb{R}^{m}$ is the state vector, $\mathbf{y} \in \mathbb{R}^{q}$ the measured output vector and $\mathbf{u} \in \mathbb{R}^{p}$ the input or control vector. $f(\cdot, \cdot, \cdot)$ and $h(\cdot, \cdot, \cdot)$ are nonlinear functions of states and inputs. Such a general model can represent a spaced discretised nonlinear system with distributed parameters, provided that a sufficiently narrow grid, influencing the state dimension $m$, is considered. In Section 3.2, the observer model for the PEMFC is developed.

\subsection{Observation problem}

As explained previously, a primary NDPO will estimate the gas concentrations profiles following model-based techniques. The PEMFC model presented in Section 2 can be computationally expensive. On the contrary, the observer is based on a model that only includes the gas flow dynamics in the gas channels on the z-direction and considers the rest of the PEMFC model as internal disturbances. In Figure 1 (bottom right) the observer modelling domain is shown. Note that it does not include the GDLs, CLs and membrane models, opposed to the simulation model (see Figure 1 (bottom left)).

As in the simulation model, in order to design the observer, a finite elements representation of the system along the z-axis is considered so that the set of PDEs representing the system dynamics can be approximated by a set of Ordinary Differential Equations (ODE). Following the state space representation in (12), the observer states are the gas species concentrations $c_{i}$, henceforth for $n$ discretisation volumes, $\mathbf{x}(t) \triangleq c_{i}\left(t, z_{1}, z_{2}, \ldots, z_{n}\right)$. The input vector includes the input molar flows $\dot{n}_{i, i n}$, therefore $\mathbf{u}(t) \triangleq \dot{n}_{i, i n}(t)$. In this paper, it is assumed that the PEMFC cathode inlet is fed with air, therefore, nitrogen is considered as a state variable and the measured outputs used to obtain the output concentrations vector $\mathbf{y}$ are expressed as

$$
\mathbf{y}(t) \triangleq\left[\begin{array}{c}
c_{H_{2}, n}^{A}(t) \\
c_{H_{2} O, n}^{A}(t) \\
c_{N_{2}, n}^{C}(t) \\
c_{O_{2}, n}^{C}(t) \\
c_{H_{2} O, n}^{C}(t)
\end{array}\right] .
$$

The computation of the output concentration vector (13) is detailed in Section 3.3.

\subsection{Observation model}

Expressing Eq. (6) in state space representation (see Eq. (12)) and taking into account that the states of the system are the concentration values, it can be written that

$$
\begin{aligned}
\dot{x}_{i}^{k}(t, z) & =\frac{\partial}{\partial z}\left(v^{k}(t, z) x_{i}^{k}(t, z)\right)-\frac{\dot{n}_{i}^{k}(t)}{\delta^{k}}, \\
v^{k}(t, z) & =-K^{k} \frac{\partial p^{k}(t, z)}{\partial z}, \\
p^{k}(t, z) & =R T^{k} \sum_{i} x_{i}^{k}(t, z),
\end{aligned}
$$

where subscript $k$ denotes the gas channel of the PEMFC, with $k \equiv A$ for the anode and $k \equiv C$ for the cathode.

The boundary conditions used are the input molar flows and pressure at the output of the gas channels. Hence, forward and backward discretisation techniques are applied to Eq. (14). Considering the boundary conditions given by [17]

$$
\begin{aligned}
v_{\text {in }} x_{i, i n} & =\dot{n}_{i, i n} \triangleq u_{i}(t), \\
p_{\text {out }} & =p_{n}=p^{a m b},
\end{aligned}
$$


and a spatial-discretisation over $n$ finite volumes, the concentration of component $i$ at volume $j$ of Eq. (14) can be expressed as

$$
\begin{aligned}
& \dot{x}_{i, j}^{k}(t)= \begin{cases}\frac{u_{i}^{k}(t)}{\Delta z}-\zeta_{1}^{k} \Psi^{k}(j)-\frac{\dot{n}_{i, j}^{k}(t)}{\delta^{k}}, & \text { if } j=1, \\
\zeta_{1}^{k}\left(\Psi^{k}(j-1)-\Psi^{k}(j)\right)-\frac{\dot{n}_{i, j}^{k}(t)}{\delta^{k}}, & \text { if } 2 \leq j \leq n-1, \\
\zeta_{1}^{k} \Psi^{k}(j-1)+\Gamma^{k}(j+1)-\frac{\dot{n}_{i, j}^{k}(t)}{\delta^{k}}, & \text { if } j=n,\end{cases} \\
& y_{i}^{k}(t)=x_{i, n}^{k},
\end{aligned}
$$

with

$$
\begin{aligned}
\Psi^{k}(j) & =x_{i, j}^{k}(t)\left(\sum_{i} x_{i, j}^{k}(t)-\sum_{i} x_{i, j+1}^{k}(t)\right), \\
\Psi^{k}(j-1) & =x_{i, j-1}^{k}(t)\left(\sum_{i} x_{i, j-1}^{k}(t)-\sum_{i} x_{i, j}^{k}(t)\right), \\
\Gamma^{k}(j+1) & =\zeta_{1}^{k} x_{i, j}^{k}(t) \sum_{i} x_{i, j}^{k}(t)+\zeta_{2}^{k} x_{i, j}^{k}(t),
\end{aligned}
$$

being $\zeta_{1}^{k}=K R T / \Delta z^{2}$ and $\zeta_{2}^{k}=K p^{a m b} / \Delta z^{2}$.

As introduced in Eq. (12), the size of the state dimension is equal to $m$. Since there are five concentrations considered in the PEMFC, the total state dimension is

$$
m=5 n,
$$

being $n$ the number of discretisation volumes considered for Eq. (16). When developing the observer, the tradeoff between discretisation refinement and mathematical complexity, i.e., the problem of defining the number of discretisation elements $n$, will be considered. Moreover, the observation problem could be further simplified if the PEMFC was fed with pure oxygen, reducing the state dimension to $m=4 n$, since the nitrogen state value would be disregarded in Eq. (16).

\subsection{Measurements}

The measurement of the gas concentrations in the last discretisation volume is not a trivial task. Gas purity sensors are expensive and in general, they operate under large response times, making them unfitting for the estimation function. However, it is possible to infer the aforementioned concentrations from other measurements present in a PEMFC system.

Knowing the output anode relative humidity and the water saturation pressure at the system temperature, it is possible to infer the water concentration value from the water partial pressure $\left(p_{n, H_{2} O}^{A}\right)$ as derived from (14c):

$$
c_{H_{2} O, n}^{A}(t)=\frac{p_{H_{2} O, n}^{A}(t)}{R T^{A}}
$$

Moreover, knowing the output pressure $\left(p_{n}^{A}\right)$ of the anode gas channel is usual in the majority of the PEMFCbased systems. From this output pressure and using (19) it is possible to obtain the partial pressure of hydrogen, since

$$
p_{n}^{A}(t)=p_{H_{2}, n}^{A}(t)+p_{H_{2} O, n}^{A}(t) .
$$

And following the same procedure as in (19), the hydrogen concentration at the end of the anode gas channel is derived.

In the cathode side there is an additional gas in comparison with the anode side. Therefore, an additional equation is needed to obtain the output concentrations. Employing a humidity sensor, the output water concentration can be obtained.

Measuring the total current demanded by the load $I(t)$, the total reacted oxygen flow rate (denoted by subscript $r$ ) is obtained [19]

$$
\dot{n}_{O_{2}, r}^{C}(t)=L_{x} L_{z} \frac{I(t)}{4 F} .
$$

The input molar flows are considered as measured variables. This is accomplished using flow meters. Knowing the reacted oxygen $(21)$ and the oxygen input molar flow $\dot{n}_{\mathrm{O}_{2}, \text { in }}^{C}$, the total output oxygen molar flow is computed

$$
\dot{n}_{O_{2}, n}^{C}(t)=\dot{n}_{O_{2}, i n}^{C}(t)-\dot{n}_{O_{2}, r}^{C}(t),
$$


which represents the flux of oxygen at the output of the cathode gas channel. Eq. (22) would introduce a certain error due to the fact that the internal dynamics of the channel are disregarded. However, this fact would only affect the observation procedure during a short transitory period which can be neglected as studied in the results section.

The oxygen concentration at the output of the cathode is obtained dividing $\dot{n}_{O_{2}, n}^{C}$ by the output gas flow velocity $v_{n}^{C}$. This velocity can be obtained applying Darcy-Weisbach principle [21]

$$
\frac{\Delta p^{C}(t)}{L_{z}}=f_{D} \frac{\rho_{\text {air }}}{2} \frac{v_{\text {out }}^{2}(t)}{D^{C}},
$$

where $\Delta p^{C}$ is the measured difference between the input and output cathode manifold pressures, $\rho_{\text {air }}$ the air density, $D^{C}$ the hydraulic diameter of the cathode gas channel and $f_{D}$ is the friction factor. For fuel cells, the flow is considered to be laminar and therefore

$$
f_{D}(t)=\frac{64}{R e(t)}
$$

being Re the Reynolds number

$$
\operatorname{Re}(t)=\frac{v_{i n}(t) D^{C}}{\nu}
$$

where $\nu$ is the kinematic viscosity of air. Eq. (23) can be rewritten as:

$$
v_{\text {out }}^{C}(t)=\sqrt{\frac{\Delta p(t)}{L} \frac{2 D}{f_{D}(t) \rho_{\text {air }}}}
$$

Therefore,

$$
c_{O_{2}, n}^{C}(t)=\frac{\dot{n}_{O_{2}, n}^{C}(t)}{v_{\text {out }}^{C}(t)} .
$$

From Eq. (14c), and knowing the output concentrations of water and oxygen, the partial pressures can be obtained. Moreover, knowing $p_{\mathrm{H}_{2} \mathrm{O}, n}^{C}, p_{\mathrm{O}_{2}, n}^{C}$ and the total output pressure $p_{n}^{C}$, the nitrogen partial pressure at the end of the cathode gas channel is obtained. Finally, the nitrogen concentration is computed using the previous values.

Henceforth, in the present paper, the following measurements are assumed to be available:

- Relative humidity at the end of both gas channels

- Pressures in the input and output manifolds both for the anode and cathode gas channels

- Input molar fluxes for all the gas species

- Temperatures in the PEMFC

- Total demanded current

The measurement of the input molar fluxes and specially the measurement of the output relative humidities imply a time delay that can affect the behaviour of the proposed estimation techniques. Specifically, relative humidity sensors work with response times of less than 5 seconds to $90 \%$ of the final value and 10 seconds for the $100 \%$ [22]. Molar flow meters perform with response times around 1 second [23].

\section{NDPO with disturbance observer}

In [15] a NDPO assuming measured disturbances was presented. In the present paper this unrealistic assumption is removed and the internal reactions and water transport terms are considered as internal disturbances and thus, their values have to be estimated.

\subsection{Primary NDPO}

Developing the model-based nonlinear observer structure proposed in [24] with the discretised observation model presented in Eq. (16) for a $n$-order nonlinear system, the observer is such that

$$
\begin{aligned}
\dot{\hat{\mathbf{x}}}(t) & =\mathbf{f}(t, \hat{\mathbf{x}}(t), \mathbf{u}(t))+\mathbf{g}(t, \hat{\mathbf{x}}(t)) \mathbf{u}_{0}(t), \\
\hat{\mathbf{y}}(t) & =\mathbf{h}(t, \hat{\mathbf{x}}(t)),
\end{aligned}
$$


where $\hat{\mathbf{x}} \in \mathbb{R}^{5 \times n}$ denotes the observed state vector and $\hat{\mathbf{y}} \in \mathbb{R}^{5}$ the observed output vector. Functions $\mathbf{f}$ and $\mathbf{h}$ include the nonlinear dynamics of the observation model presented in Eq. (16). Moreover, $\mathbf{u} \in \mathbb{R}^{p}$ is the input vector, where $p$ is the number of input molar fluxes of the PEMFC gas species, which is equal to five in this paper. Finally, $\mathbf{u}_{0} \in \mathbb{R}^{5}$ is the corrective input term for each one of the output concentrations species values. The corrective input term is a function of the output observation error $\mathbf{e}_{y}$, defined as the difference between the estimated output $\hat{\mathbf{y}}(28 \mathrm{~b})$ and the measured vector (13) as expressed by

$$
\mathbf{e}_{y}(t)=\hat{\mathbf{y}}(t)-\mathbf{y}(t)
$$

where $\mathbf{e}_{y} \in \mathbb{R}^{5}$.

The implementation details of Eq. (28) are given in [15]. The aim of this paper is to remove the assumptions that were considered in the previous research and improve the NDPO considering the reaction rates and water molar flows $\dot{n}_{i}$ as disturbances of the observer. Disturbance estimation (Section 4.2) and back-stepping differentiation (Section 5) techniques are proposed in the following sections to achieve the aforementioned reaction and water transport disturbances estimation.

\subsection{Disturbance observer}

The observation-oriented model from Eq. (16) can be expressed as a function of the dynamics of the plant, inputs and unmatched disturbances:

$$
\begin{aligned}
\dot{x}_{i, j}^{k}(t) & =f\left(x_{i, j}^{k}(t)\right)+g_{1, i}^{k} u^{k}+g_{2, i}^{k} d_{i, j}^{k}(t), \\
y_{i}^{k}(t) & =x_{i, n}^{k}(t)
\end{aligned}
$$

where $d_{i, j}^{k}(t) \triangleq \dot{n}_{i, j}^{k}(t) \in \mathbb{R}^{5 \times n}$ denotes the reaction and water transport terms vector, which is considered as unknown and unmeasurable. Moreover, $f$ represents the nonlinear dynamics of the PEMFC system, $g_{1, i}^{k}$ and $g_{2, i}^{k}$ are the inputs and disturbance gains vectors of the $i$-th gas species. The gains vectors, following Eq. (16), are expressed as

$$
\begin{aligned}
g_{1, i}^{k} & =\frac{1}{\Delta z}[1,0, \ldots, 0] \\
g_{2, i}^{k} & =-\frac{1}{\delta^{k}}[1,1, \ldots, 1]
\end{aligned}
$$

for the $n$ discretisation volumes.

To estimate the nonlinear disturbances $d_{i, j}^{k}$ in (30), a NDOB [25] is introduced

$$
\begin{aligned}
\dot{p^{k}} & =-l^{k} g_{2, i}^{k}\left(x_{i, j}^{k}(t)\right) p^{k}-l^{k}\left[p_{1}(t)+p_{2}(t)+p_{3}(t)\right], \\
\hat{d}_{i, j}^{k}(t) & =p^{k}+l^{k} x_{i, j}^{k}(t),
\end{aligned}
$$

with

$$
\begin{aligned}
& p_{1}(t)=g_{2, i}^{k}\left(x_{i, j}^{k}(t)\right) l^{k} x_{i, j}^{k}(t), \\
& p_{2}(t)=f\left(x_{i, j}^{k}(t)\right) \\
& p_{3}(t)=g_{1, i}^{k}\left(x_{i, j}^{k}(t)\right) u^{k}
\end{aligned}
$$

where $\hat{d}^{k}$ is the estimation of the disturbance and $p^{k}$ represents the internal nonlinear dynamics of the NDOB. The gain is represented by $l^{k}$. The final topology of the NDPO with the NDOB extension is shown in Figure 2.

\section{HOSM observer}

The HOSM observer proposed in this paper is based on quasi-continuous control techniques as in [26]. The approach is faster and more accurate than the NDPO with NDOB introduced in Section 4 as it will be shown in the results section of this paper. Its hierarchical structure to recover the full gas concentrations profile of the PEMFC is shown in Figure 3. From the value of the output states (13), a back-stepping procedure is applied to recover the full state profile of the concentrations. In the NDPO with NDOB extension the state and disturbance estimation were independent. Contrarily, the proposed HOSM observer integrates both operations in the same topology (see Figure 3). 


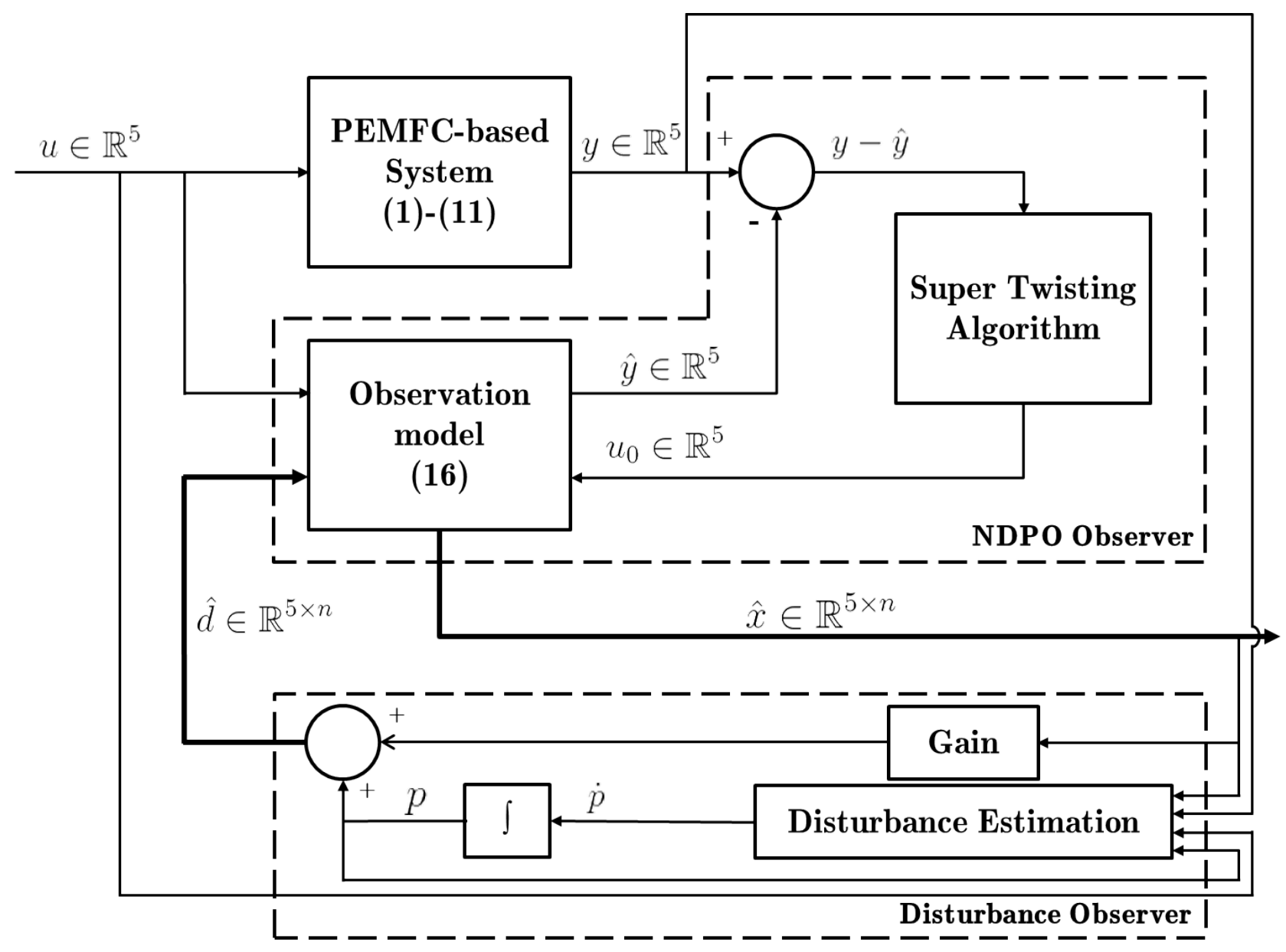

Figure 2: NDPO with NDOB extension

\subsection{HOSM back-stepping algorithm}

To track the output concentrations vector (13) and drive the state estimation to zero in a finite time, a novel back-stepping observation algorithm is developed in this paper, rewriting the HOSM control strategy proposed in [26] for state estimation purposes. The novelty of the technique is that it is the first time that such HOSM algorithm is used for robust state estimation in distributed parameters nonlinear systems. The HOSM relative degree is equal to $n$.

The procedure follows an $r$-step algorithm, where $r \in[1,2, \ldots, n]$, as shown in Algorithm 1.

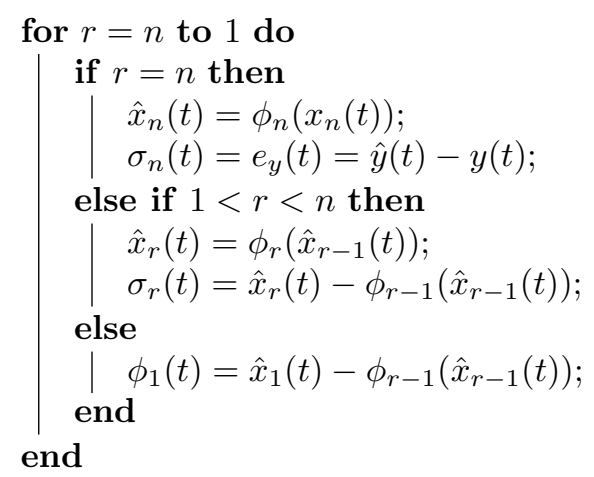

Algorithm 1: HOSM back-stepping algorithm

The quasi-continuous term that has to drive the observation error (29) to zero is included in the $\phi_{r}\left(\hat{x}_{r}\right)$ terms [26], which are defined as

$$
\phi_{r}\left(\hat{x}_{r}, t\right)=\mathbf{B}_{r}\left(\hat{x}_{r}, t\right)^{-1}\left\{-f_{r}+u_{r}\right\}
$$

where $\mathbf{B}_{r}$ is the product between the output transformation $\sigma_{n}$ and the input vector field $u \triangleq \dot{n}_{i, i n}^{k} \in \mathbb{R}^{5}$. Moreover, $f_{r}$ is the nonlinear dynamics of Eq. (16). An $r$-sliding homogeneous corrective action $u_{r}$ [27, 28] has 
to be designed for $r<n$

$$
u_{r}(t)=-\alpha_{r} \Psi\left(\sigma, \dot{\sigma}, \ldots, \sigma^{r-1}\right)
$$

with $\sigma_{1}=\hat{x}_{1}-\phi\left(\hat{x}_{1}\right)$. The computation of the derivatives $\sigma, \dot{\sigma}, \ldots, \sigma^{r-1}$ is made using robust differentiators with finite-time convergence [29].

Until now, the output error in Eq. (29) has been shown to be eliminated. Moreover, the minimisation of the state observation error $x-\hat{x}$ will be studied in Section 6 . As is the case of the NDPO with disturbance observer, the back-stepping algorithm produces an accumulative estimation error since the full state profile is being estimated from the five output measurements. Nevertheless, as it will be analysed in Section 6, the robustness of the proposed method produces satisfactory results with almost negligible error.

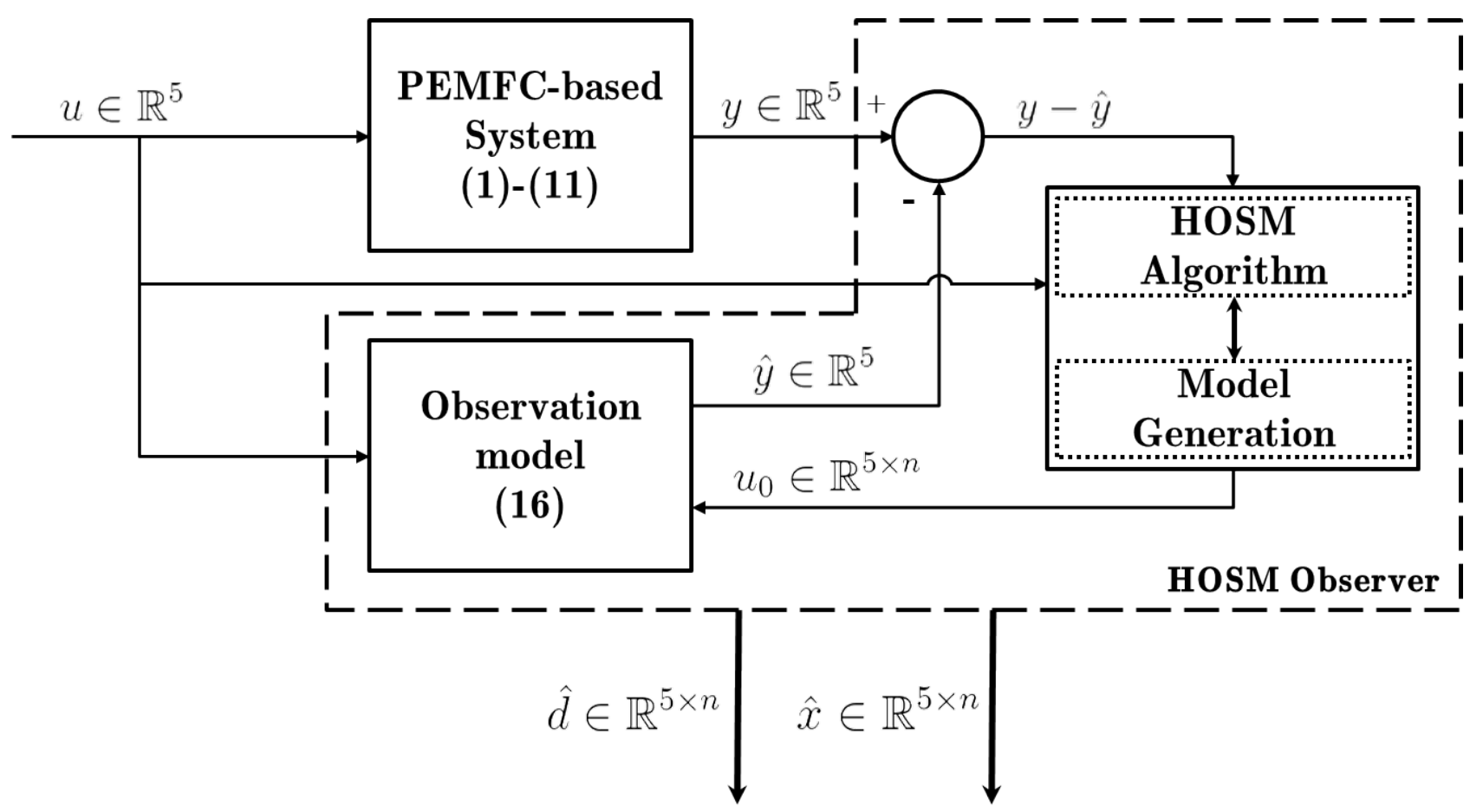

Figure 3: HOSM observer topology

\section{Results}

The two developed observers are compared in the present section through a numerical simulation analysis under the same given conditions.

\subsection{Numerical implementation}

The vector $\mathbf{x}_{\mathbf{0}} \in \mathbb{R}^{5 \times n}$ defines the initial state for all simulations and it has a dimension equal to five times the number of discretisation volumes, denoting the five gas species present in the gas channels for each one of the volumes. For the shown results, the mesh for the simulation model and the observer consists of 5 elements equally distributed. Both observers are initialised with the initial observer state vector $\hat{\mathbf{x}}_{\mathbf{0}}=\mathbf{0} \in \mathbb{R}^{5 \times n}$. Simulations have been carried out using Simulink for MATLAB ${ }^{\circledR}$ R2011a (32 bits), running in a PC Intel ${ }^{\circledR}$ Core $^{\text {TM }}$ i7-3770 at $3.40 \mathrm{GHz}$ with $8 \mathrm{~GB}$ of RAM.

\subsection{Simulation scenario}

To test the dynamic performance of the developed observers, a New European Driving Cycle (NEDC) current profile is going to be applied to the PEMFC. The NEDC profile is shown in Figure 4 . The observers do not have the NEDC current profile available in advance in order to test their performance on the event of unknown external disturbances such as drastic current changes. The PEMFC is operated with anode and cathode stoichiometric rates of 1.3 , a constant inlet pressure of 1.23 bar, a PEMFC temperature of $353 \mathrm{~K}$ and a constant humidity of $50 \%$ for both gas channel imputs. 


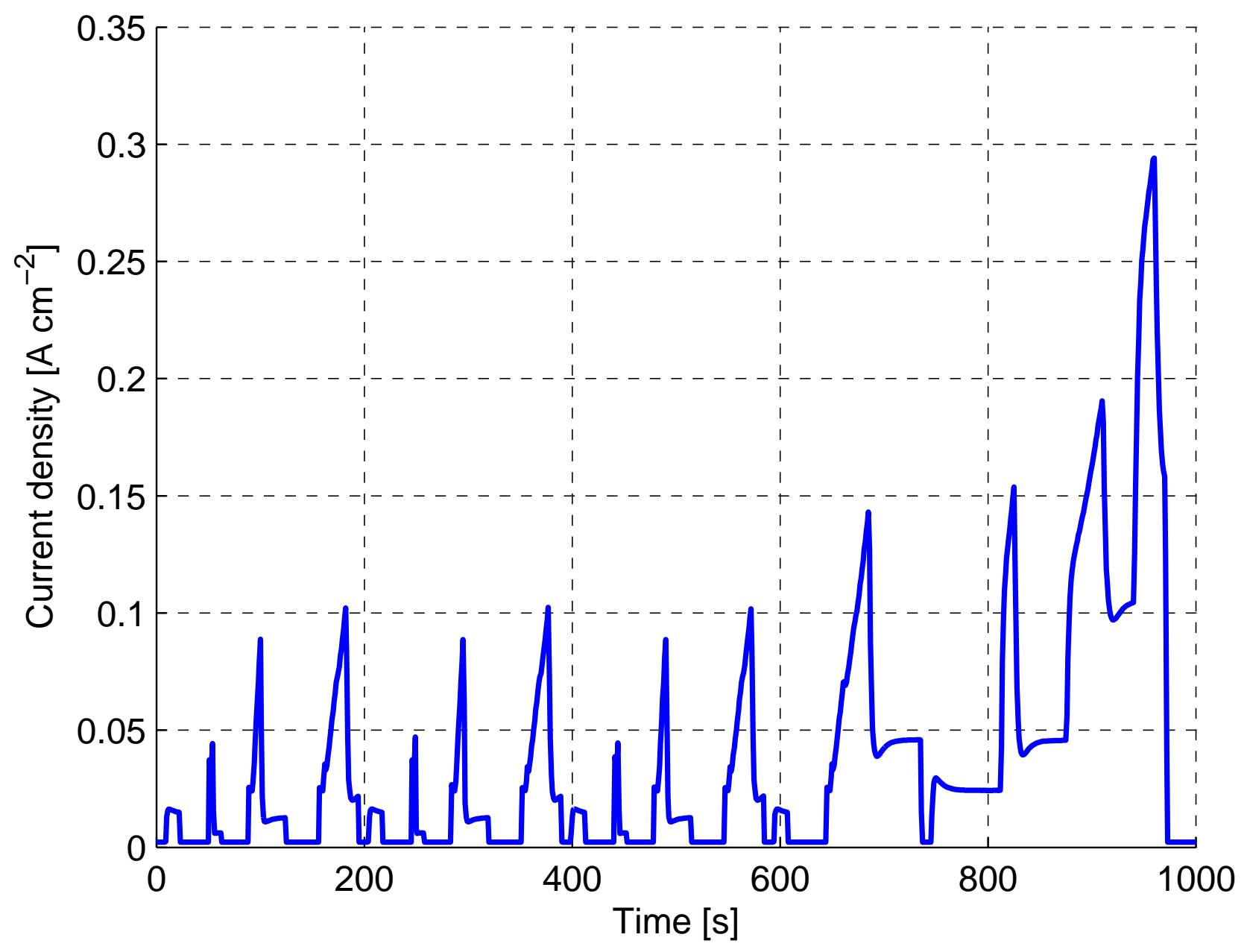

Figure 4: Demanded current density (NEDC profile) [30] 


\subsection{Performance indicator}

The state observation error of the $i$-th gas species through the $n$ discretisation volumes is written as

$$
\mathbf{e}_{i}(t)=\hat{\mathbf{x}}_{i}(t)-\mathbf{x}_{i}(t)=\left[\begin{array}{c}
e_{i, 1}(t) \\
e_{i, 2}(t) \\
\vdots \\
e_{i, n}(t)
\end{array}\right]
$$

To compare the proposed observers, a performance indicator for the state observation error (36) is introduced. In particular, for this paper, the mean absolute error (MAE)

$$
M A E(t)=\frac{1}{n} \sum_{i}^{5} \sum_{j=1}^{n}\left|e_{i, j}(t)\right|=\frac{1}{n} \sum_{i}^{5} \sum_{j=1}^{n}\left|\hat{x}_{i, j}(t)-x_{i, j}(t)\right|
$$

is proposed. Note that $(37)$ is defined for all the discretised volumes, which considers all the $n$ volumes in the anode and cathode gas channels, not only the output volumes. It also considers all the $i$ reactants. As mentioned, this error is computed by comparing the state values at a given moment of the simulation with the observed ones from each one of the observer structures. The total value is computed considering the simulation time $t_{\text {sim }}$

$$
M A E_{T}=\int_{0}^{t_{s i m}} M A E(t) d t
$$

\subsection{Results and discussion}

After the simulation of the case study presented in Section 6.2, the total MAEs for the HOSM observer and the NDPO with NDOB extension over a 1000 s simulation time are

$$
\begin{aligned}
M A E_{T, H O S M} & =1.54 \%, \\
M A E_{T, N D O B} & =1.79 \% .
\end{aligned}
$$

Both observers perform satisfactorily under the simulation conditions, nevertheless, the average MAE of the HOSM observer is lower and its performance its superior to the NDOB approach.

The observation of the anode gas channel concentrations in the middle point of the PEMFC are shown in Figure 5 and the cathode gas channel concentrations in the middle point of the PEMFC are shown in Figure 6. As explained The observation of the gas concentration species is done for all the discretisation volumes, however Figures 5 and 6 only show the middle point of the anode and cathode gas channels. This point is of special interest because it is practically impossible to install sensors in this section of the fuel cell and commercial systems are not prepared to obtained measures inside the PEMFC. It can be seen that the HOSM observer detects possible local starvation points faster than the NDOB observer. This information can be used in a closed-loop control topology in order to avoid possible degradation of the PEMFC.

As shown in Figures 5 and 6, the observation of the gas species (represented as dashed lines) is performed properly in a simulation environment, even in the presence of steep changes in the current profile (see Figure 4). These current variations create a fast response in the concentration values than can result in local fuel and oxidant starvation points. Without the use of state observation techniques it is not possible to detect these starvation points and therefore, apply actions that can improve or avoid the situation. For the present paper, the operating conditions (input molar flows) have remained constant as presented in Section 6.2, since the focus of the study is on the development of the observation techniques.

\subsection{Observation of the disturbances}

An inherent characteristic of the proposed observers is the estimation of the reaction and water transport terms (flow rates from the membrane to the channels) in parallel with the state estimation. These values can be used to map the current density distribution along the PEMFC without the need to use segmented cells or internal sensors.

According to the simulation scenario of the previous section, the reaction and water terms are obtained using the HOSM approach to the observation problem. In Figure 7, the estimated hydrogen, oxygen and water transport terms for the case study in the middle of the PEMFC are presented for the first 80 seconds of the case study. At each side of the membrane, positive values mean that the transport occurs from the gas channels towards the membrane in the $y$-direction. When the sign of the molar transport term is negative it means that the flow travels towards the gas channels, as is the case of the water transport terms during some parts of the simulation. 

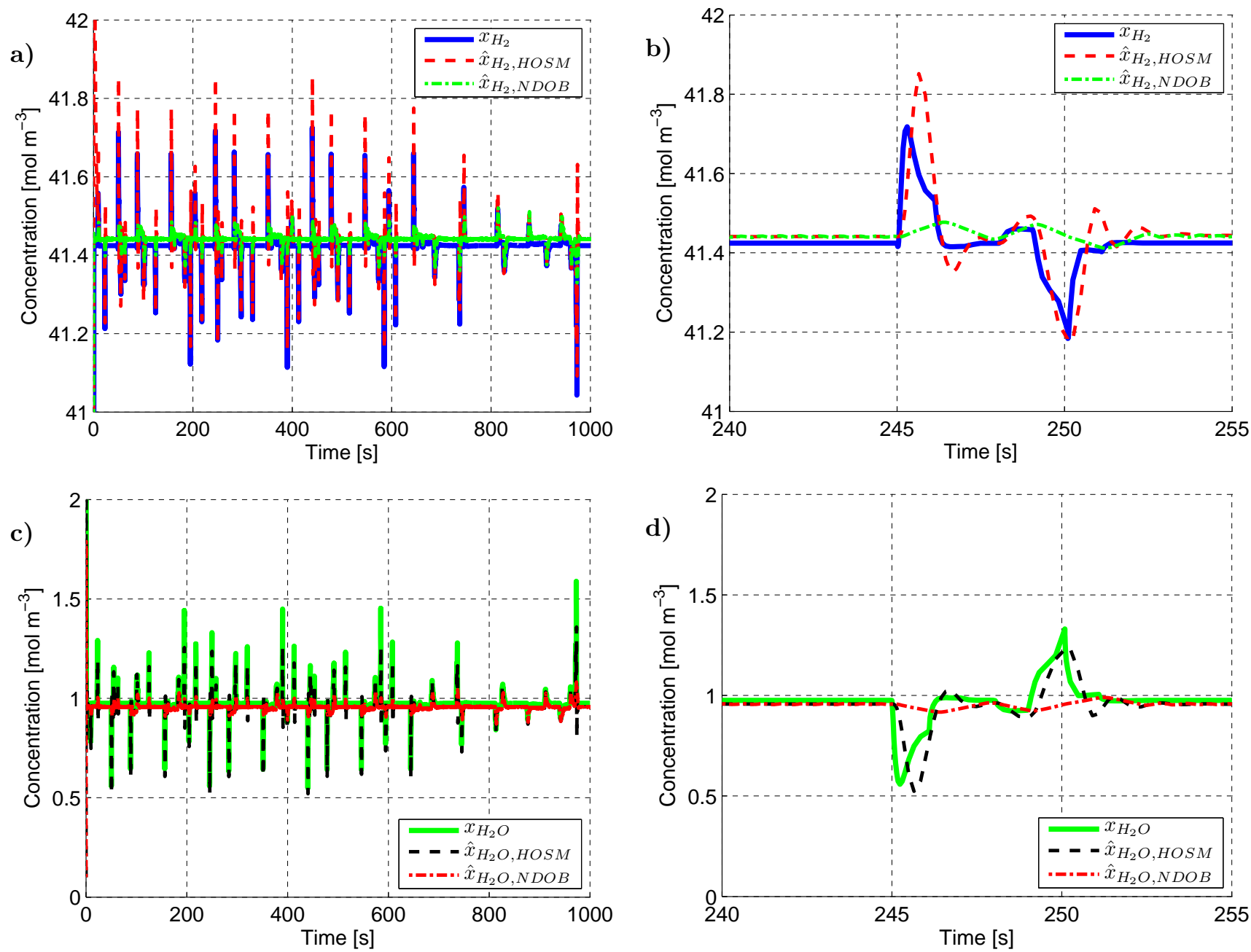

Figure 5: Observation of hydrogen ( $\mathrm{a}$ and $\mathrm{b}$ ) and water (c and $\mathrm{d}$ ) concentrations in the middle point of the anode gas channel 

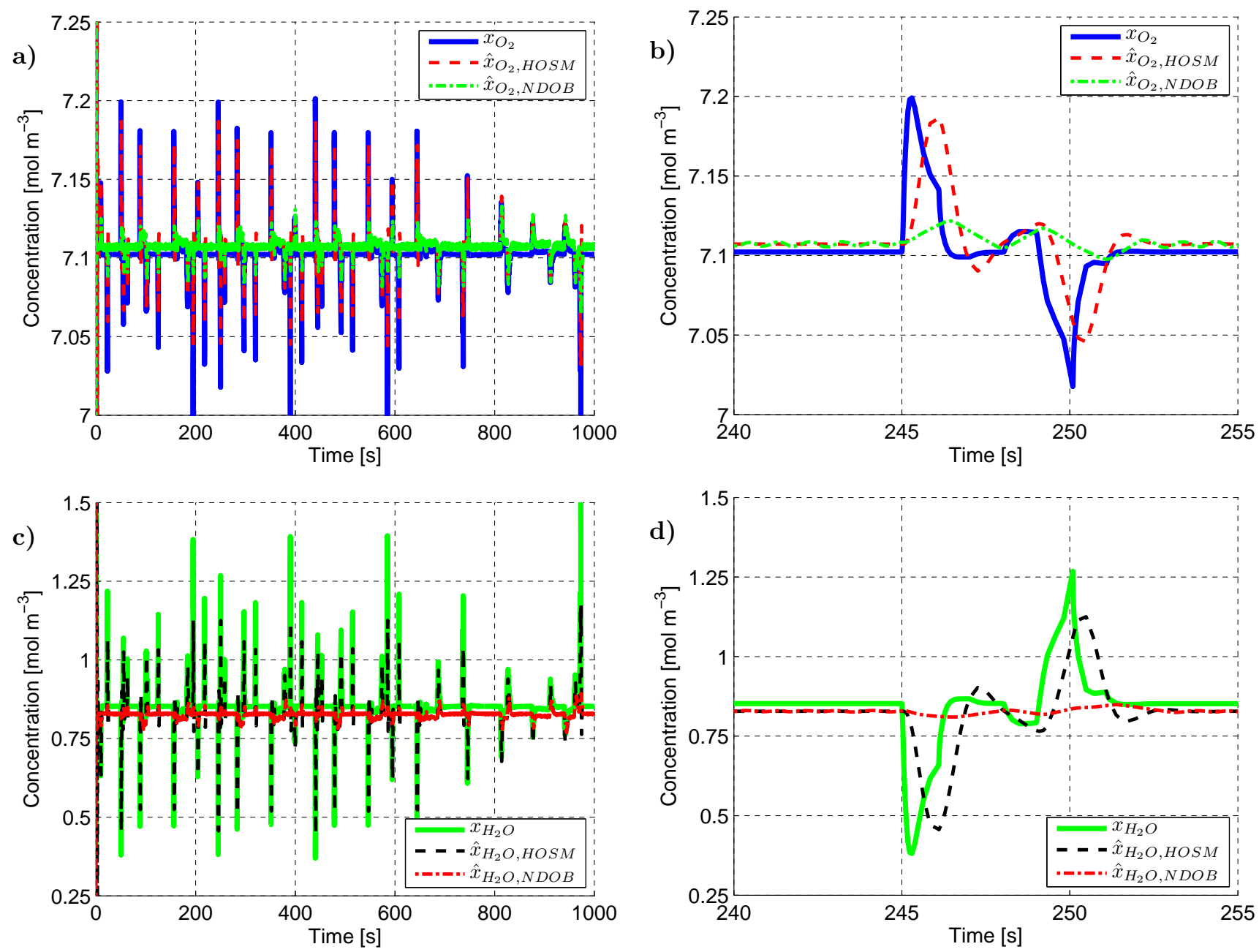

Figure 6: Observation of oxygen ( $a$ and $b$ ) and water ( $\mathrm{c}$ and $\mathrm{d}$ ) concentrations in the middle point of the cathode gas channel 

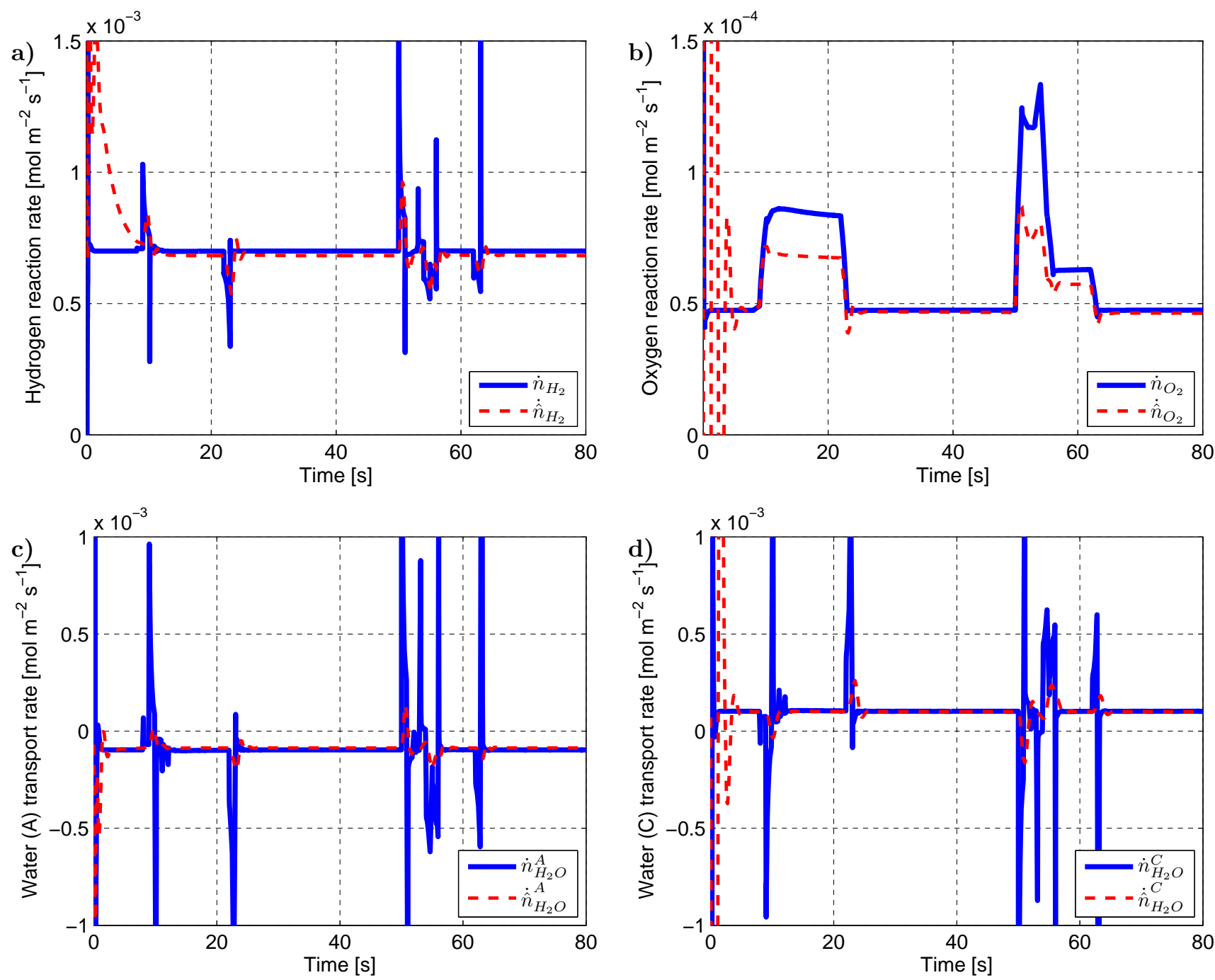

Figure 7: Hydrogen (a), oxygen (b) and water (c and d) transport terms in the middle point of the anode and cathode gas channels 
Using the hydrogen reaction rate showed in Figure 7(a) and considering Eqs. (2), (3) and (4), it is possible to obtain the exchange current density $i_{0}$ along the z-direction of the PEMFC, obtaining the full current profile using only the available measurements.

The oxygen reaction rate in the middle volume of the cathode gas channel is shown in Figure 7(b). As expected, it follows the current profile demand and given its positive value, it is a flow from the channel towards the membrane in the y-direction of the PEMFC.

Water transports behave similarly as depicted in Figure $7(\mathrm{c})$ and (d). The negative sign in Figure $7(\mathrm{c})$ indicates that water is travelling from the membrane towards the anode gas channel. The positive values in Figure $7(\mathrm{~d})$ display that the water is flowing from the cathode gas channel towards the membrane. The water transport dynamics are driven by the membrane model presented in Section 2.3.4.

\subsection{Robustness analysis}

The robustness for the NDPO without disturbance observation was studied in [15]. In this section, the robustness for the HOSM observer is analysed. Particularly, to test the proper convergence with model uncertainties, a temperature gradient from 0 to $10 \mathrm{~K}$ between the cathode gas channel and cathode catalyst layer is considered. As it is shown in Figure 8, the observer is able to track the state under the presence of this particular model uncertainties.

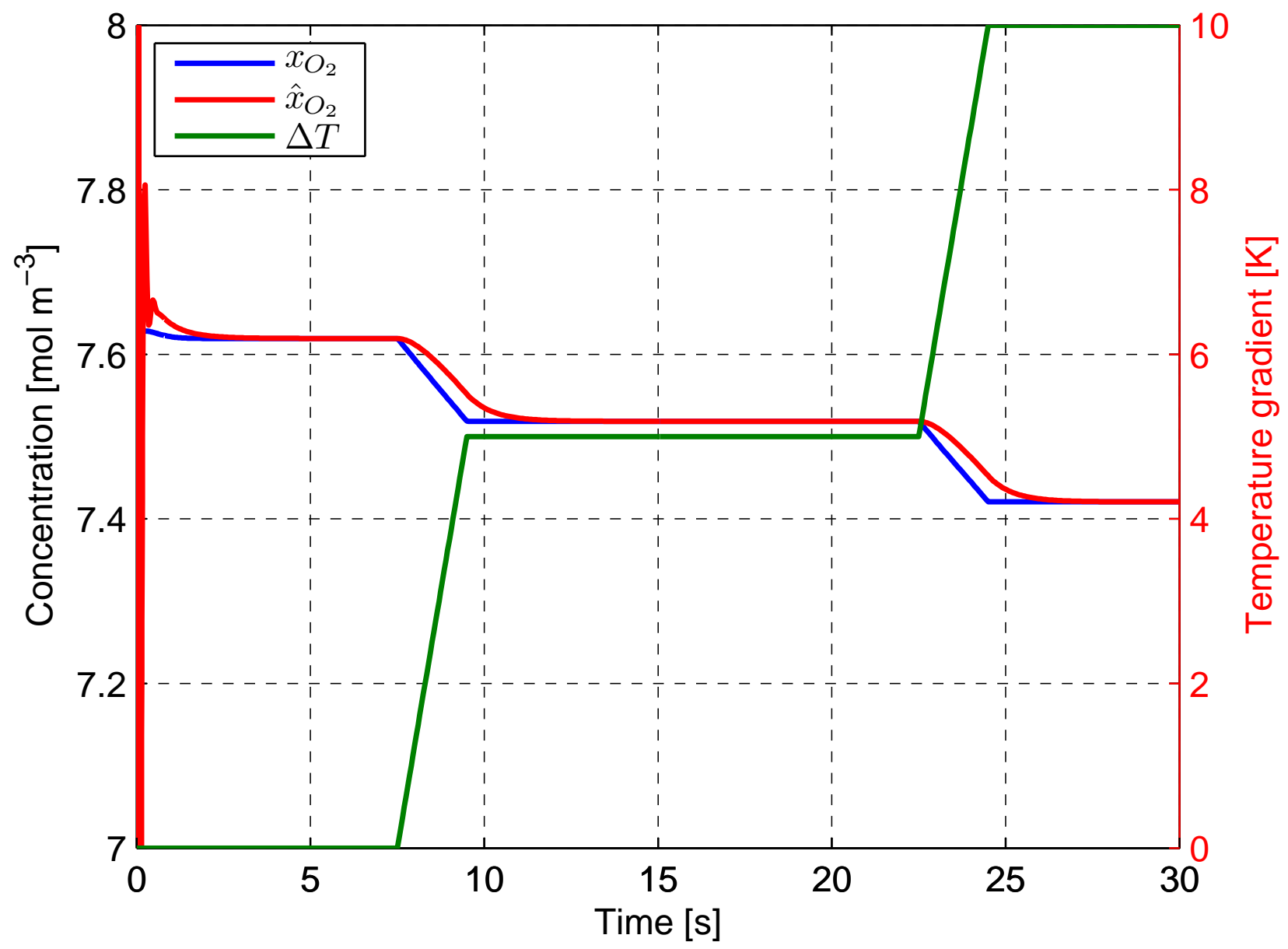

Figure 8: HOSM oxygen estimation in the cathode gas channel in the presence of temperature gradients between the cathode gas channel and catalyst layers

\section{Conclusions}

In this paper, two nonlinear estimation solutions are proposed to observe the full state gas concentration profiles in a PEMFC. Besides, the two estimation strategies allow for estimating the reaction and water transport terms for a distributed parameter PEMFC model. One approach is the NDPO with NDOB extension and the other is the HOSM observer. The two strategies have been compared and their performance evaluated, obtaining satisfactory observation results for a particular simulation framework. The MAE along the anode and cathode gas channels shows the better performance of the HOSM over the disturbance estimation procedure. 
A total of five discretisation volumes for the distributed parameter model have been considered to carry on the simulations. From the point of view of the observers the increase of the number of discretisation volumes introduces mathematical complexity when synthesising the aforesaid observers. It is necessary to achieve a tradeoff between the required level of detail of the recovered state information and the mathematical complexity of the observers. Although, with the five discretisation configuration the information that can be extracted from the simulations is quite satisfactory. Nevertheless, future research will be aimed at generalising the approach to higher numbers of discretisation points in order to increase the detail of the solution.

It is forthcoming the inclusion of the proposed nonlinear observers in a closed-loop control topology to implement output-feedback techniques that take advantage of the full nonlinear estimation of the internal states. Furthermore, the observer can also be integrated with diagnosis tools to display the current PEMFC health status.

\section{Acknowledgements}

This work has been partially funded by the Spanish national project MICAPEM (ref. DPI2015-69286-C32R), the Regione Autonoma della Sardegna project CRP-7733 and the the European project PUMA-MIND (ref. SPI-JTI-FCH 303419).

\section{References}

[1] M. Bitzer, M. Zeitz, Design of a nonlinear distributed parameter observer for a pressure swing adsorption plant, Journal of Process Control 12 (4) (2002) 533-543.

[2] J. Luna, A. Husar, M. Serra, State observers design for PEMFC systems, in: Ibero-American Conference on Hydrogen and Fuel Cells 2014, Bellaterra, 2014, pp. 144-147.

[3] M. Hinaje, I. Sadli, J. Martin, P. Thounthong, S. Raël, B. Davat, Online humidification diagnosis of a PEMFC using a static DC-DC converter, international Journal of Hydrogen Energy 34 (6) (2009) 27182723.

[4] G. Tian, S. Wasterlain, I. Endichi, D. Candusso, F. Harel, X. François, M.-C. Péra, D. Hissel, J.-M. Kauffmann, Diagnosis methods dedicated to the localisation of failed cells within PEMFC stacks, Journal of Power Sources 182 (2) (2008) 449-461.

[5] R. Petrone, Z. Zheng, D. Hissel, M.-C. Péra, C. Pianese, M. Sorrentino, M. Becherif, N. Yousfi-Steiner, A review on model-based diagnosis methodologies for PEMFCs, International Journal of Hydrogen Energy 38 (17) (2013) 7077-7091.

[6] M. Bavarian, M. Soroush, I. G. Kevrekidis, J. B. Benziger, Mathematical modeling, steady-state and dynamic behavior, and control of fuel cells: A review, Industrial \& Engineering Chemistry Research 49 (17) (2010) 7922-7950.

[7] K. Thanapalan, G.-P. Liu, J. G. Williams, B. Wang, D. Rees, Review and analysis of fuel cell system modelling and control, International Journal of Computer Aided Engineering and Technology 1 (2) (2009) 145-157.

[8] J. Luna, C. Ocampo-Martinez, M. Serra, Nonlinear predictive control for the concentrations profile regulation under unknown reaction disturbances in a fuel cell anode gas channel, Journal of Power Sources 282 (2015) 129-139.

[9] N. Yousfi-Steiner, P. Moçotéguy, D. Candusso, D. Hissel, A review on polymer electrolyte membrane fuel cell catalyst degradation and starvation issues: Causes, consequences and diagnostic for mitigation, Journal of Power Sources 194 (1) (2009) 130-145.

[10] H. Görgün, M. Arcak, F. Barbir, An algorithm for estimation of membrane water content in PEM fuel cells, Journal of Power Sources 157 (1) (2006) 389-394.

[11] H. Görgün, F. Barbir, M. Arcak, A voltage-based observer design for membrane water content in PEM fuel cells, in: Proceedings of the American Control Conference, IEEE, 2005, pp. 4796-4801.

[12] M. Arcak, H. Görgün, L. Pedersen, S. Varigonda, A nonlinear observer design for fuel cell hydrogen estimation, IEEE Transactions on Control Systems Technology 12 (1) (2004) 101-110.

[13] I. Kazmi, A. Bhatti, S. Iqbal, A nonlinear observer for PEM fuel cell system, in: IEEE 13th International Multitopic Conference, IEEE, 2009, pp. 1-6. 
[14] A. Pilloni, A. Pisano, E. Usai, Observer based air excess ratio control of a PEM fuel cell system via high order sliding mode, IEEE Transactions on Industrial Electronics 62 (8) (2015) 5236-5246.

[15] J. Luna, A. Husar, M. Serra, Nonlinear distributed parameter observer design for fuel cell systems, International Journal of Hydrogen Energy 40 (34) (2015) 11322-11332.

[16] A. Levant, Quasi-continuous high-order sliding-mode controllers, IEEE Transactions on Automatic Control 50 (11) (2005) 1812-1816.

[17] M. Mangold, A. Bück, R. Hanke-Rauschenbach, Passivity based control of a distributed PEM fuel cell model, Journal of Process Control 20 (3) (2010) 292-313.

[18] W. Neubrand, Modellbildung und simulation von elektronen membranverfahren, Ph.D. thesis, Universitat Stuttgart (1999).

[19] F. Barbir, PEM fuel cells: theory and practice, Academic Press, 2012.

[20] L. Mejlbro, The complete solution of Fick's second law of diffusion with time-dependent diffusion coefficient and surface concentration, Durability of Concrete in Saline Environment (1996) 127-158.

[21] P. A. Tipler, G. Mosca, Physics for scientists and engineers, Macmillan, 2007.

[22] Met One 083E relative humidity sensor, http://www.metone.com/docs/083e_datasheet.pdf.

[23] Bronkhorst EL-FLOW, http://www.bronkhorst.com/en/products/gas_flow_meters_and_ controllers/elflow_select/.

[24] J. Davila, L. Fridman, A. Pisano, E. Usai, Finite-time state observation for non-linear uncertain systems via higher-order sliding modes, International Journal of Control 82 (8) (2009) 1564-1574.

[25] J. Yang, S. Li, X. Yu, Sliding-mode control for systems with mismatched uncertainties via a disturbance observer, IEEE Transactions on Industrial Electronics 60 (1) (2013) 160-169.

[26] A. Estrada, L. Fridman, Quasi-continuous HOSM control for systems with unmatched perturbations, Automatica 46 (11) (2010) 1916-1919.

[27] W. Perruquetti, J. Barbot, Sliding mode control in engineering, CRC Press, 2002.

[28] S. K. Spurgeon, Sliding mode observers: a survey, International Journal of Systems Science 39 (8) (2008) $751-764$.

[29] A. Levant, Higher-order sliding modes, differentiation and output-feedback control, International Journal of Control 76 (9-10) (2003) 924-941.

[30] M. Mayur, S. Strahl, A. Husar, W. G. Bessler, A multi-timescale modeling methodology for pemfc performance and durability in a virtual fuel cell car, International Journal of Hydrogen Energy 40 (46) (2015) $16466-16476$. 